\title{
Total Laparoscopic Hysterectomy for Diagnosis and Treatment of Cervical Cystic Lesions: A Case Series
}

\author{
Tohru Morisada* (1), Kohsei Tanaka, Tomoko Iijima, Azumi Nishizawa, Hiroshi Nishio, \\ Yusuke Kobayashi, Masaru Nakamura, Shigenori Hayashi, Fumio Kataoka, Takashi Iwata, \\ Daisuke Aoki
}

Department of Obstetrics and Gynecology, Keio University School of Medicine, Tokyo, Japan

Email: ${ }^{\star}$ morisada@a7.keio.jp

How to cite this paper: Morisada, T., Tanaka, K., Iijima, T., Nishizawa, A., Nishio, H., Kobayashi, Y., Nakamura, M., Hayashi, S., Kataoka, F., Iwata, T. and Aoki, D. (2020) Total Laparoscopic Hysterectomy for Diagnosis and Treatment of Cervical Cystic Lesions: A Case Series. Open Journal of Obstetrics and Gynecology, 10, 357-364. https://doi.org/10.4236/ojog.2020.1030033

Received: February 12, 2020

Accepted: March 9, 2020

Published: March 12, 2020

Copyright $\odot 2020$ by author(s) and Scientific Research Publishing Inc. This work is licensed under the Creative Commons Attribution International License (CC BY 4.0).

http://creativecommons.org/licenses/by/4.0/

\begin{abstract}
Objective: Cervical cystic lesions are often observed in gynecological clinical practice. Many are caused by benign diseases such as lobular endocervical glandular hyperplasia (LEGH), and it is important to differentiate these lesions from those due to malignant diseases such as minimal deviation adenocarcinoma (MDA). In cases in which fertility preservation is not a concern, the final pathology is often confirmed by hysterectomy. To investigate the feasibility and safety of laparoscopic surgery for cervical cystic lesions, we retrospectively examined cases in which total laparoscopic hysterectomy (TLH) was performed for diagnosis and treatment. Methods: The subjects were 28 women who underwent TLH from April 2012 to March 2017 at Keio University Hospital. The pre- and post-operative courses of these patients were examined. Results: The average age was 49.4 (range 39 - 65) years and the mean body mass index was $21.7(16.8-30.5) \mathrm{kg} / \mathrm{m}^{2}$. The average operation time was 159 (101 - 314) min and the average bleeding volume was $106.8(0-600) \mathrm{g}$. There were no severe perioperative complications. The average hospital stay was $8.4(7-14)$ days. In postoperative pathological diagnosis, 13 cases had benign diseases such as Nabot cysts, there were 14 cases of LEGH, and one patient was diagnosed with MDA. In the MDA case, additional bilateral salpingo-oophorectomy and pelvic lymph node dissection were performed at a later date. No metastasis was observed in these excised specimens. Conclusions: Our results suggest that TLH for cervical cystic lesions can be performed safely. However, it is important to consider the TLH indication before surgery based on the possibility of malignant disease.
\end{abstract}

\section{Keywords}

Lobular Endocervical Glandular Hyperplasia, Minimum Deviation 
Adenocarcinoma, Total Laparoscopic Hysterectomy, Uterine Cervix

\section{Introduction}

Cervical cystic lesions are common in gynecological clinical practice. As a cervical cystic lesion, benign diseases including lobular endocervical glandular hyperplasia (LEGH) are difficult to distinguish from malignant diseases such as minimal deviation adenocarcinoma (MDA) [1]. MDA is an extremely well-differentiated mucinous adenocarcinoma with only minimal histological deviation from normal cervical glands, and its prognosis is poor due to resistance to surgical treatment and chemotherapy [2] [3] [4]. Due to their characteristics, MDA lesions are often misdiagnosed as benign lesions of the uterine cervix by cytology or in small-sized superficial biopsies [5].

LEGH was first reported by Nucci et al. as a benign lesion characterized by lobular proliferation of glands lined by endocervical mucin-producing epithelial cells [6]. Both MDA and LEGH present as a multicystic mass in the uterine cervix in methods such as magnetic resonance imaging (MRI), which makes differential diagnosis of cervical cystic lesions difficult [7] [8]. Moreover, these lesions can extend to the deep cervical canal. Therefore, proper preoperative diagnosis may not be obtained even after performing cervical conization.

Since the expected concordance rate between pre- and post-operative diagnoses of cervical cystic lesions is relatively low, the final pathology is confirmed by hysterectomy in cases for which fertility preservation is not important. However, in general, minimally invasive surgery is needed for these lesions. Here, we retrospectively investigated the feasibility and safety of total laparoscopic hysterectomy (TLH) for diagnosis and treatment of cervical cystic lesions.

\section{Materials and Methods}

\subsection{Study Design and Patient Population}

A retrospective case analysis was performed in 28 women who underwent TLH for cervical cystic lesions from April 2012 to March 2017 at Keio University Hospital. Preoperative imaging findings, cytological examination, and diagnosis based on histology (cervical biopsy or cervical conization); surgical procedure; postoperative pathologic diagnosis; and clinical course were examined from medical records. Cytology results were reported in accordance with the 2001 Bethesda system. Histological diagnosis was conducted based on the General Rules for Clinical and Pathological Management of Uterine Cervical Cancer (3rd edition).

\subsection{Surgical Procedure}

After insertion of the primary trocar and laparoscope, $\mathrm{CO}_{2}$ pneumoperitoneum was established at $10 \mathrm{mmHg}$ and three suprapubic cannulas were introduced. 
Surgery was performed by the diamond type 4-port method after an accurate abdominal and pelvic inspection. Given the possibility of malignant diseases, the uterus was removed using an extra-fascicular procedure. The entire specimen was removed via the vagina, and the vaginal cuff was sutured laparoscopically with absorbent thread. The surgical time was defined as the period from the umbilical incision to closure of abdominal wounds. Blood loss in surgery was determined by subtracting the total volume in the suction bottle from the irrigation fluid volume.

\subsection{Ethical Approval}

This retrospective study was conducted in accordance with the Declaration of Helsinki and was approved by the IRB of Keio University Hospital (approval number: 20150197).

\section{Results}

The average age of the 28 patients was 49.4 (range 39 to 65 ) years and the mean body mass index was $21.7(16.8-30.5) \mathrm{kg} / \mathrm{m}^{2}$ (Table 1$)$. As a clinical symptom, the $28.6 \%(8 / 28)$ cases had watery discharge before surgery. The average operation time was $159(101-314) \mathrm{min}$ and the average bleeding volume was $106.8(0$ - 600) $\mathrm{ml}$. The average hospitalization period was 8.4 (7 - 14) days (Table 2) and no cases had severe complications.

A comparison of preoperative diagnosis of LEGH by MRI and postoperative pathological diagnosis is shown in Table 3. In preoperative pelvic MRI, there were 6 cases diagnosed as benign cervical lesions, 17 cases as LEGH, and 5 in which the possibility of malignant lesions could not be completely excluded. Of the 17 cases diagnosed as LEGH, 10 were found to be LEGH in postoperative pathological results, giving a correct preoperative diagnostic rate by MRI of $58.8 \%(10 / 17)$.

Postoperative pathological diagnosis indicated 13 cases of benign diseases such as Nabot cysts and 14 cases of LEGH. Minimal deviation adenocarcinoma after surgery was found in one case (Table 4). In the MDA case, additional

Table 1. Characteristics of the patients.

\begin{tabular}{ccc}
\hline Item & Average & Range \\
\hline Age (years) & 49.4 & $39-65$ \\
BMI $\left(\mathrm{kg} / \mathrm{m}^{2}\right)$ & 21.7 & $16.8-30.5$ \\
\hline
\end{tabular}

Table 2. Operation and clinical course.

\begin{tabular}{ccc}
\hline Item & Average & Range \\
\hline Operation time (min) & 159 & $101-314$ \\
Blood loss (g) & 106.8 & $0-600$ \\
Hospital stay (days) & 8.4 & $7-14$ \\
\hline
\end{tabular}


Table 3. Postoperative pathologic diagnosis of cases with a preoperative LEGH diagnosis by MRI.

\begin{tabular}{cc}
\hline Diagnosis & Number of cases \\
\hline Benign diseases & 6 \\
LEGH & 10 \\
Malignant lesions & 1 \\
\hline
\end{tabular}

Table 4. Histological examination of the specimens.

\begin{tabular}{cc}
\hline Histological examination & Number of cases \\
\hline Benign diseases & 13 \\
LEGH & 14 \\
MDA & 1 \\
\hline
\end{tabular}

bilateral salpingo-oophorectomy and pelvic lymph node dissection were performed at a later date. No metastasis was observed in these excised specimens. To date, no recurrence of the lesion has been observed in the subjects examined, including this case.

In cases in which diagnostic conization was performed prior to TLH, this procedure showed benign diseases such as chronic cervicitis in 5 cases and LEGH in 7 cases. A comparison of histopathological diagnosis using conization specimens and pathological diagnosis of tissue specimens collected by TLH is shown in Table 5. The concordance rate between these two methods was $85 \%$ $(11 / 13)$.

\section{Discussion}

Cervical cystic lesions are common clinical features in gynecology. These lesions may be pathologically benign diseases such as LEGH, but may also reflect malignant diseases such as MDA. Thus, the preoperative diagnostic accuracy is important, but this is often difficult, including with use of diagnostic cervical conization. Cytological examination and punch biopsy also have limited efficacy for differential diagnosis of these lesions [9].

Diagnostic imaging using MRI has been reported to be effective [10]. LEGH frequently shows the following findings on MRI: lesions at a high position in the cervix, a so-called "cosmos sign" that shows a collection of relatively large cysts around small cysts and solid parts on the luminal side of the cervix, and clearly demarcated lesion margins. In contrast, MDA often appears on MRI as a solid tumor with an indistinct border showing a higher signal than cervical stroma on a T2-weighted image, invasive growth around the periphery, and relatively small cysts that are widely distributed in the cervix [11].

A correct diagnostic rate of $87.8 \%$ (36/41 cases) was obtained for LEGH based on the typical Cosmos sign on MRI [11], whereas in the current study this rate for LEGH in preoperative MRI was $58.8 \%$ (10/17 cases). In MDA, cytology 
Table 5. Comparison of histopathological diagnosis of cone-excised specimens and TLH specimens.

\begin{tabular}{ccccc}
\hline Case & Age & $\begin{array}{c}\text { Conization } \\
\text { specimen }\end{array}$ & $\begin{array}{c}\text { TLH } \\
\text { specimen }\end{array}$ & $\begin{array}{c}\text { Validity of } \\
\text { preoperative diagnosis }\end{array}$ \\
\hline 1 & 46 & Chronic cervicitis & Chronic cervicitis & Adequate \\
2 & 54 & Chronic cervicitis & LEGH & Under-diagnosis \\
3 & 51 & CIN3 & Chronic cervicitis & Adequate \\
4 & 52 & LEGH & LEGH & Adequate \\
5 & 48 & LEGH & Chronic cervicitis & Adequate \\
6 & 50 & LEGH & LEGH & Adequate \\
7 & 45 & LEGH & LEGH & Adequate \\
8 & 44 & LEGH & LEGH & Adequate \\
9 & 49 & Chronic cervicitis & MDA & Under-diagnosis \\
10 & 52 & Chronic cervicitis & Benign cystic lesion & Adequate \\
11 & 50 & LEGH & LEGH & Adequate \\
12 & 44 & Chronic cervicitis & Benign cystic lesion & Adequate \\
13 & 50 & LEGH & LEGH & Adequate \\
\hline
\end{tabular}

shows mainly AGC and adenocarcinoma, but the positive rate in cytology is not as high as expected. Furthermore, both MDA and LEGH may coexist with each other and with other malignant or benign lesions. For example, Okuyama et al. [12] reported two cases of cervical adenocarcinoma in situ arising from lobular endocervical hyperplasia, together with retrospective cytological findings. Therefore, careful evaluation is required in reaching a preoperative diagnosis.

For cervical cystic lesions, it is often difficult to make a preoperative differential diagnosis by cytology or histology, and cervical conization may be performed for this purpose. Regarding the accuracy, a concordance rate of $92.3 \%(12 / 13$ cases) has been reported for pathological diagnosis using conization and hysterectomy [11], indicating that diagnostic conization is useful for assessing the primary lesion. In the current study, 13 cases underwent diagnostic conization and $85 \%(11 / 13)$ were correctly diagnosed preoperatively. In hysterectomy after conization, there are general concerns about adverse effects such as postoperative infection [13]. However, in our patients, there was no significant difference in the mean operation time (144.5 vs. $171.4 \mathrm{~min}$ ) and blood loss (116.1 vs. 98.7 g) between cases with and without conization (Table A1).

In this study, we experienced two cases in which the pathological diagnosis by hysterectomy was more advanced than that in conization specimens. Because of the limitations of preoperative diagnosis with imaging and conization, TLH may be increasingly indicated for cystic cervical lesions in clinical practice. In our hospital, the TLH technique is essentially based on an extra-fascial procedure, given the possibility of neoplastic lesions. We note that minimally invasive surgery for cervical malignant tumors has been suggested to be inferior to open 
surgery in terms of oncologic outcome [14] [15]. Thus, given this point, careful consideration should be given to the indication of TLH for cervical cystic lesions.

There are some limitations to the study, including the retrospective analysis of medical records from a single institute, which might have resulted in some bias in case selection. A prospective multicenter study with a larger sample size is needed to verify our results.

\section{Conclusion}

Our findings suggest the safety of total laparoscopic hysterectomy for cervical cystic lesions. However, LEGH may coexist with malignant lesions such as MDA, and care with the indication for TLH is needed because of possible coexisting and de novo adenocarcinoma.

\section{Competing Interests}

The authors declare that there are no conflicts of interest regarding the publication of this article.

\section{Author's Contributions}

Tohru Morisada: Substantially contributed to the study conception and design as well as the acquisition and interpretation of the data and drafting the manuscript.

Kosei Tanaka, Tomoko Iijima and Azumi Nishizawa: contributed to the acquisition of data.

Hiroshi Nishio, Yusuke Kobayashi, Masaru Nakamura, Shigenori Hayashi, and Fumio Kataoka: contribution to the analysis and interpretation of data.

Takashi Iwata and Daisuke Aoki: Drafting manuscript and approved the final version of the manuscript towards publication.

\section{Acknowledgements}

We are grateful to all the patients and co-investigators for their assistance with the study.

\section{References}

[1] Ding, D.C., Chu, T.Y. and Hsu, Y.H. (2016) Minimal Deviation Adenocarcinoma of the Uterine Cervix: A Case Report. Tzu-Chi Medical Journal, 28, 79-81. https://doi.org/10.1016/j.tcmj.2014.10.001

[2] Mikami, Y. and McCluggage, W.G. (2013) Endocervical Glandular Lesions Exhibiting Gastric Differentiation: An Emerging Spectrum of Benign, Premalignant, and Malignant Lesions. Advances in Anatomic Pathology, 20, 227-237. https://doi.org/10.1097/PAP.0b013e31829c2d66

[3] Mikami, Y. (2020) Gastric-Type Mucinous Carcinoma of the Cervix and Its Precursors: Historical Overview. Histopathology, 76, 102-111.

https://doi.org/10.1111/his.13993 
[4] Talia, K.L. and McCluggage, W.G. (2018) The Developing Spectrum of Gastric-Type Cervical Glandular Lesions. Pathology, 50, 122-133. https://doi.org/10.1016/j.pathol.2017.09.009

[5] Itoh, K., Toki, T., Shiohara, S., Oguchi, O., Konishi, I. and Fujii, S. (2000) A Comparative Analysis of Cross Sectional Imaging Techniques in Minimal Deviation Adenocarcinoma of the Uterine Cervix. BJOG: An International Journal of Obstetrics and Gynaecology, 107, 1158-1163. https://doi.org/10.1111/j.1471-0528.2000.tb11117.x

[6] Nucci, M.R., Clement, P.B. and Young, R.H. (1999) Lobular Endocervical Glandular Hyperplasia, Not Otherwise Specified: A Clinicopathologic Analysis of Thirteen Cases of a Distinctive Pseudoneoplastic Lesion and Comparison with Fourteen Cases of Adenoma Malignum. The American Journal of Surgical Pathology, 23, 886-891. https://doi.org/10.1097/00000478-199908000-00005

[7] Ohya, A., Asaka, S., Fujinaga, Y. and Kadoya, M. (2018) Uterine Cervical Adenocarcinoma Associated with Lobular Endocervical Glandular Hyperplasia: Radiologic-Pathologic Correlation. Journal of Obstetrics and Gynaecology Research, 44, 312-322. https://doi.org/10.1111/jog.13528

[8] Tsuboyama, T., Yamamoto, K., Nakai, G., Yamada, T., Fujiwara, S., Terai, Y., Ohmichi, M. and Narumi, Y. (2015) A Case of Gastric-Type Adenocarcinoma of the Uterine Cervix Associated with Lobular Endocervical Glandular Hyperplasia: Radiologic-Pathologic Correlation. Abdominal Imaging, 40, 459-465.

https://doi.org/10.1007/s00261-014-0323-6

[9] Ando, H., Miyamoto, T., Kashima, H., Takatsu, A., Ishii, K., Fujinaga, Y. and Shiozawa, T. (2016) Usefulness of a Management Protocol for Patients with Cervical Multicystic Lesions: A Retrospective Analysis of 94 Cases and the Significance of GNAS Mutation. Journal of Obstetrics and Gynaecology Research, 42, 1588-1598. https://doi.org/10.1111/jog.13083

[10] Doi, T., Yamashita, Y., Yasunaga, T., Fujiyoshi, K., Tsunawaki, A., Takahashi, M., Katabuchi, H., Tanaka, N. and Okamura, H. (1997) Adenoma Malignum: MR Imaging and Pathologic Study. Radiology, 204, 39-42.

https://doi.org/10.1148/radiology.204.1.9205220

[11] Takatsu, A., Shiozawa, T., Miyamoto, T., Kurosawa, K., Kashima, H., Yamada, T., Kaku, T., Mikami, Y., Kiyokawa, T., Tsuda, H., Ishii, K., Togashi, K., Koyama, T., Fujinaga, Y., Kadoya, M., Hashi, A., Susumu, N. and Konishi, I. (2011) Preoperative Differential Diagnosis of Minimal Deviation Adenocarcinoma and Lobular Endocervical Glandular Hyperplasia of the Uterine Cervix: A Multicenter Study of Clinicopathology and Magnetic Resonance Imaging Findings. International Journal of Gynecological Cancer, 21, 1287-1296. https://doi.org/10.1097/IGC.0b013e31821f746c

[12] Okuyama, R., Hashimoto, H., Miura, T., et al. (2017) Two Cases of Adenocarcinoma in Situ Arising in Lobular Endocervical Glandular Hyperplasia Indicating Localization of Mucin on the Cluster Surface as an Early Cytological Finding of Malignant Transformation. Diagnostic Cytopathology, 45, 842-847. https://doi.org/10.1002/dc.23740

[13] Phongnarisorn, C. and Srisomboon, J. (2007) Surgical Morbidity Associated with Total Laparoscopic Hysterectomy in Women with Prior Diagnostic Excision of the Cervix. The Journal of Obstetrics and Gynaecology Research, 33, 519-523. https://doi.org/10.1111/j.1447-0756.2007.00565.x

[14] Ramirez, P.T., Frumovitz, M., Pareja, R., Lopez, A., Vieira, M., Ribeiro, R., Buda, A., Yan, X., Shuzhong, Y., Chetty, N., Isla, D., Tamura, M., Zhu, T., Robledo, K.P., 
Gebski, V., Asher, R., Behan, V., Nicklin, J.L., Coleman, R.L. and Obermair, A. (2018) Minimally Invasive versus Abdominal Radical Hysterectomy for Cervical Cancer. The New England Journal of Medicine, 379, 1895-1904.

https://doi.org/10.1056/NEJMoa1806395

[15] Pennington, K.P., Urban, R.R. and Gray, H.J. (2019) Revisiting Minimally Invasive Surgery in the Management of Early-Stage Cervical Cancer. Journal of the National Comprehensive Cancer Network, 17, 86-90.

https://doi.org/10.6004/jnccn.2018.7263

\section{Abbreviations}

LEGH-Lobular Endocervical Glandular Hyperplasia

MDA-Minimum Deviation Adenocarcinoma

TLH-Total Laparoscopic Hysterectomy

\section{Supplementary}

Table A1. Operation data and clinical course of TLH with or without prior conization.

\begin{tabular}{ccc}
\hline Item & TLH after conization & TLH without conization \\
\hline Operation time (min) & $144.5(101-197)$ & $171.4(116-314)$ \\
Blood loss $(\mathrm{g})$ & $116.1(0-600)$ & $98.7(0-250)$ \\
Hospital stay (days) & $7.9(7-10)$ & $8.7(7-14)$ \\
\hline
\end{tabular}

